\title{
Diacronie
}

Studi di Storia Contemporanea

$N^{\circ} 16,4 \mid 2013$

Le monarchie nell'età dei nazionalismi

\section{Gianluca Scroccu, Il partito al bivio. Il PSI dall'opposizione al governo (1953-1963)}

Jacopo Perazzoli

\section{(2) OpenEdition}

Edizione digitale

URL: http://journals.openedition.org/diacronie/964

DOI: 10.4000/diacronie.964

ISSN: 2038-0925

Editore

Association culturelle Diacronie

Notizia bibliografica digitale

Jacopo Perazzoli, « Gianluca Scroccu, II partito al bivio. II PSI dall'opposizione al governo (1953-1963) », Diacronie [Online], № 16, 4 | 2013, documento 15, Messo online il 01 décembre 2013, consultato il 24 septembre 2020. URL : http://journals.openedition.org/diacronie/964 ; DOI : https://doi.org/10.4000/ diacronie.964 


\section{Diacronie}

N. 16 | 4|2013 Le monarchie nell'età dei nazionalismi

15/

\section{RECENSIONE:}

\section{Gianluca SCROCCU, Il partito al bivio. Il PSI}

\section{dall'opposizione al governo (1953-1963), Roma, Carocci, 2011, 358 pp.}

a cura di Jacopo PERAZZOLI *

Gianluca Scroccu, ricercatore presso il Dipartimento di Studi Storici, Geografici e Artistici dell'Università di Cagliari, si è occupato approfonditamente dell'evoluzione del socialismo italiano nel secondo dopoguerra. Soffermatosi sui singoli protagonisti di quella stagione con i volumi La passione di un socialista: Sandro Pertini e il PSI dalla Liberazione agli anni del centro-sinistra ${ }^{1}$ e Alla ricerca di un socialismo possibile. Antonio Giolitti dal PCI al $P S I^{2}$, ha in seguito rielaborato il tema su un piano più generale come dimostra quest'opera, frutto della sua tesi di dottorato3.

Il partito al bivio si inserisce tra i tentativi della storiografia degli ultimi anni di riconsiderare l'intera vicenda del PSI nel corso degli anni Cinquanta. Un decennio non più ritenuto un periodo buio fortemente segnato dalla stagione frontista 4 , ma analizzato come una fase in cui il partito di Nenni stava iniziando a mutare pelle, in vista della nascita del centro-sinistra grazie ad una maggioranza con i democristiani, i socialdemocratici e i repubblicani. In questa corrente storiografica rientrano a pieno titolo i volumi di Paolo Mattera, Il partito inquieto. Organizzazione, passioni e politica dei socialisti italiani dalla Resistenza al miracolo economico5; di Giovanni Scirocco,

\footnotetext{
${ }^{1}$ SCROCCU, Gianluca, La passione di un socialista: Sandro Pertini e il PSI dalla Liberazione agli anni del centro-sinistra, Manduria, Lacaita, 2008.

2 SCROCCU, Gianluca, Alla ricerca di un socialismo possibile. Antonio Giolitti dal PCI al PSI, Roma, Carocci, 2012.

3 SCROCCU, Gianluca, Il partito al bivio. Il PSI dall'opposizione al governo (1953-1963), Roma, Carocci, 2011, p. 15.

4 È il caso, ad esempio, del lavoro di DI SCALA, Spencer, Il socialismo italiano da Turati a Craxi. Il socialismo italiano visto dagli USA, Milano, SugarCo, 1990.

5 MATTERA, Paolo, Il partito inquieto. Organizzazione, passioni e politica dei socialisti italiani dalla Resistenza al miracolo economico, Roma, Carocci, 2004.
} 
Politique d'abord: il PSI, la guerra fredda e la politica internazionale (1948-1957)6; e di Maria Margherita Scotti, Da sinistra. Intellettuali, Partito socialista italiano e organizzazione della cultura (1953-1960)7. Tuttavia, mentre queste opere sono finalizzate a fare luce sul fronte della politica interna ed estera, sull'ambito culturale e sull'attivismo socialista con lo scopo di ridimensionare la conclamata subalternità del PSI al Partito Comunista, il libro di Scroccu analizza un decennio fondamentale per la storia dei socialisti italiani: dalla sconfitta della "legge truffa" del 1953 fino alla scissione della sinistra socialista nel dicembre 1963 in seguito all'ingresso a pieno titolo del PSI nella squadra di governo guidata da Aldo Moro.

L'autore porta a termine questo ambizioso progetto attraverso uno studio della «storia dei socialisti italiani alla luce dei nuovi modelli interpretativi della storiografia italiana che hanno consentito la collaborazione tra indagine storica e scienze sociali» ${ }^{8}$. Prestando la giusta attenzione al linguaggio usato dai socialisti nel corso di questi anni, considerato una sorta di termometro attraverso cui misurare e valutare il mutamento di pelle del PSI, Scroccu ha scelto di mettere al centro della sua analisi le tematiche dell'identità, della mobilitazione, delle forme di propaganda e dell'organizzazione interna, «a partire dalle relazioni spesso conflittuali tra le scelte dei gruppi dirigenti e l'attività della base e dei simpatizzanti» ${ }^{9}$. Tematiche decisive, nell'ottica dell'autore, per «comprendere i mutamenti dei partiti politici italiani durante gli anni del miracolo economico» ${ }^{10}$.

Grazie ad uno scavo archivistico condotto non solo nelle istituzioni che conservano il materiale dei soggetti politici in questione - la Fondazione Lelio Basso, la Fondazione Pietro Nenni, la Fondazione di Studi Storici Filippo Turati, la Fondazione Istituto Gramsci - ma anche nel fondo del Ministero dell'Interno relativo ai partiti politici conservato presso l'Archivio Centrale dello Stato, l'autore ha potuto dar vita a quattro intensi capitoli. Nel primo, Un frontismo possibile 1953-1957, sono ripercorsi i cambiamenti di rotta del PSI che, da una posizione più propriamente filo-comunista, incominciava a ricercare la propria autonomia ancora prima della rottura con il PCI in seguito ai drammatici eventi del 1956. Parallelamente a questa ricostruzione Scroccu, citando quanto sostenuto da Vittorio Foa in Questo Novecento ${ }^{11}$, evidenzia le carenze

\footnotetext{
${ }^{6}$ SCIROCCO, Giovanni, Politique d'abord: il PSI, la guerra fredda e la politica internazionale (1948-1957), Milano, UNICOPLI, 2010.

7 SCOTTI, Maria Margherita, Da sinistra. Intellettuali, Partito socialista italiano e organizzazione della cultura (1953-1960), Roma, Ediesse, 2011.

${ }^{8}$ SCROCCU, Gianluca, Il partito al bivio, cit., p. 7.

9 Ibidem.

${ }^{10}$ Ibidem.

${ }^{11}$ FOA, Vittorio, Questo Novecento, Torino, Einaudi, 1996, p. 253.
} 
socialiste nell'analisi della società degli anni cinquanta. A tali insufficienze il PSI, se realmente intenzionato a diventare una forza di governo, avrebbe dovuto giocoforza porre un rimedio attraverso la trasformazione della militanza, da ricercare non più esclusivamente tra le file della classe operaia, ma anche nei ceti medi che, proprio da questi anni, incominciarono a diventare oggetto di interesse da parte del partito di Nenni. Ma ciò, come osserva lucidamente l'autore, non sarebbe avvenuto «in tempi brevi» ${ }^{2}$.

Il secondo capitolo, Il biennio incerto (1958-59), si apre con la descrizione della campagna elettorale per le politiche del 1958 in cui il PSI ottenne un positivo 14,23\%, per chiudersi con la fase successiva al congresso di Napoli del gennaio 1959, nel corso del quale iniziavano a farsi sempre più forti le divergenze tra gli autonomisti, che avevano ottenuto la maggioranza negli organismi dirigenti, e la sinistra interna di Vecchietti e Valori, che era contraria alla collaborazione governativa del PSI con la Democrazia Cristiana. Particolarmente degno di nota in questa sezione è l'approfondimento dedicato all'attenzione del partito di Nenni verso le dinamiche e le novità presenti all'interno del corpo elettorale che rappresentava uno dei punti più rilevanti della strategia politica socialista. A causa della comparsa del fenomeno del consumismo, che era una diretta conseguenza del dirompente sviluppo dell'economia, stavano mutando i valori sedimentati nella società italiana. Per essere in grado di ampliare il proprio bacino di preferenze, dunque, era inevitabile che il PSI affrontasse il cambiamento radicale nello stile di vita e nei comportamenti individuali degli italiani di quegli anni. Nondimeno, i limiti dell'azione socialista nell'affrontare questa spinosa questione sono notevoli, come sottolinea l'autore, poiché il PSI si dimostrava ancora incapace di mettere da parte la propria interpretazione marxista della società.

Oltre a prendere in considerazione le posizioni socialiste di fronte al governo Tambroni e allo sbocciare di quelle che Moro definì le "convergenze parallele", nel terzo capitolo, Il magnifico e complicato 196o, Scroccu si sofferma sui contenuti della proposta politica del partito di Nenni, cercando di sottolineare, da un lato, le ambiguità che ancora contraddistinguevano la linea socialista; e dall'altro, di dare il giusto risalto anche alle nuove interpretazioni che iniziavano ad apparire nei documenti teorici formulati dal PSI. In questo senso l'autore, grazie ad un attento studio della documentazione disponibile, ha evidenziato come i socialisti iniziassero a superare la concezione del declino inarrestabile del capitalismo a livello mondiale e a pensare ad una critica più costruttiva del mercato. «Il persistere del richiamo ad una coscienza di classe si accompagnava [...] all'esortazione verso una scelta precisa in materia di

${ }^{12}$ SCROCCU, Gianluca, Il partito al bivio, cit., p. 65 . 
consumi e di investimenti attraverso un indefinito metodo democratico superiore all'arbitrio delle concentrazioni di potere monopolistico» ${ }^{13}$.

L'autore conclude il suo ragionamento nell'ultimo capitolo, Gli anni di costruzione del centro-sinistra (1961-1963), in cui risaltano due tematiche principali. La prima è rappresentata dalla messa a punto, per mano di Riccardo Lombardi e di Antonio Giolitti, della piattaforma economico-politico-programmatica che avrebbe poi contraddistinto l'azione dei socialisti una volta entrati a pieno titolo nella maggioranza di governo e che si sarebbe dovuta basare «sull'evoluzione del ruolo dello Stato [...] in materia di pianificazione pubblica, [...] ispirandosi ai principi di una politica democratica legata a tematiche come le riforme di struttura e la programmazione» ${ }^{14}$. Il continuo contrasto tra la maggioranza autonomista e la minoranza di sinistra - la seconda tematica - è illustrata grazie ad una puntuale citazione dei discorsi tenuti da Nenni e Vecchietti nel corso del congresso di Milano del 1961 e durante l'assise di Roma del 1963. In questa circostanza la frattura tra le due correnti era ormai insanabile. Alla decisione di Nenni di seppellire definitivamente l'immagine classista del PSI, tratteggiando il profilo di un soggetto politico che ora ambiva ad abbracciare tutto il mondo del lavoro e tutte le sue categorie, e filo-occidentale, accettando la NATO e gli obblighi che da essa derivavano, corrispondeva la volontà di Vecchietti di confermare la bontà della dimensione unitaria del movimento operaio, che avrebbe dovuto condurre le proprie lotte senza abdicare alla sua storia e, soprattutto, alla sua prospettiva di classe. Scroccu accompagna il lettore sino agli istanti precedenti la fuoriuscita della sinistra interna socialista, considerata il frutto di «una mancanza di maturità [del PSI] che ne aveva indebolito la forza» 15 ed evidenziando come la situazione interna del Partito socialista, proprio nel momento in cui stava per entrare a pieno titolo nella maggioranza di governo insieme a democristiani, socialdemocratici e repubblicani, fosse drammaticamente preoccupante per una forza politica con ambizioni governative. Sempre di fronte al complesso ingresso del PSI nelle fila del governo, Scroccu non dimentica di fare luce anche sulle difficoltà interne alla maggioranza autonomista, evidenziando le differenze tra la concezione di Nenni, che, «da parte sua, era convinto che il "rivoluzionario" accesso alla famigerata "stanza dei bottoni” potesse giustificare le infinite mediazioni»16, cui i socialisti dovevano sottostare, e la posizione di Lombardi che, al contrario, era meno disposto a venire incontro alla volontà della DC e, soprattutto, della corrente dorotea, di ridurre l'impatto riformista del governo. Proprio

${ }^{13}$ Ibidem, pp. 265-266.

14 Ibidem, p. 277.

15 Ibidem, p. 349.

${ }^{16}$ Ibidem, p. 329. 
le difficoltà dei socialisti, osserva giustamente l'autore, erano una costante nella storia della sinistra italiana:

Quella che ha portato sovente le sue componenti a dividersi di fronte alle domande che nascevano dalla società, per l'incapacità di trovare soluzioni unitarie che potessero ricomporre in una sintesi le proposte e le aspirazioni tanto dei gruppi dirigenti quanto dei militanti ${ }^{17}$.

Vi sono poi per lo meno tre spunti ulteriori che caratterizzano l'opera di Scroccu. Innanzitutto una costante attenzione nei confronti delle ripercussioni a livello locale delle decisioni compiute dagli organismi dirigenti nazionali. Esemplare, in questo senso, è quanto accaduto in Sardegna in seguito al congresso di Venezia del 1957. Basandosi sulla relazione del prefetto di Cagliari, l'autore annota l'apparire dei malumori nei confronti del gruppo dirigente autonomista, che aveva sostituito il gruppo di sinistra legato alla figura di Emilio Lussu: i risultati dell'assise nel capoluogo veneto «avevano [...] generato nell'isola un certo smarrimento tra la base [...] in cui si erano visti molti dirigenti vicini alla linea di Nenni attaccare Lussu per il suo atteggiamento filo-PCI, ritenendolo addirittura incompatibile con la militanza socialista» ${ }^{18}$.

Un'altra riflessione innovativa presentata in Il partito al bivio è quella nei confronti dei Movimento Giovanile Socialista, rinato, dopo la scissione di Palazzo Barberini del 1947, come ufficio della direzione, specializzato nelle politiche giovanili19. Anche se risultò centrale per il PSI recuperare la partecipazione dei giovani all'elaborazione politica, Scroccu non manca di osservare come il movimento giovanile sia stato un elemento frenante all'innovazione del partito poiché tra i suoi iscritti e rappresentanti era ancora molto forte il richiamo dell'URSS. La «zavorra» ${ }^{20}$, infatti, si presentò in qualsiasi circostanza in cui i socialisti tentarono di allontanarsi dall'Unione Sovietica. Di fronte al dramma del 1956, ad esempio, la segreteria del MGS celebrò le conquiste del regime ungherese. Sul fronte interno, i giovani socialisti erano propensi a confermare l'unità d'azione con il PCI. Ça va sans dire che anche questi assunti

\footnotetext{
17 Ibidem, p. 350.

${ }^{18}$ Ibidem, p. 134.

19 URL: <http://www.pertini.it/turati/a_mgs.html> [consultato il 22 agosto 2013]. Sul tema si veda anche ORSINA, Giovanni, QUAGLIARIELLO, Gaetano (a cura di), La formazione della classe politica in Europa, Manduria, Lacaita, 2000, p. 196 et seq.; DOGLIANI Patrizia, Storia dei giovani, Milano, Bruno Mondadori, 2003, pp. 181-182.

20 L'autore usa proprio questa espressione per definire il Movimento Giovanile Socialista. Si veda SCROCCU, Gianluca, Il partito al bivio, cit., p. 189.
} 
costituirono un ostacolo da superare per il gruppo autonomista sulla rotta dell'innovazione.

In ultimo, è ugualmente interessante il breve spunto comparativo attraverso cui Scroccu illustra la difficoltà dei socialisti italiani nell'interpretare e nel reagire ai cambiamenti della società degli ultimi anni cinquanta. Il metro di paragone è la Socialdemocrazia tedesca e, nello specifico, quanto adottato dalla SPD nel congresso di Bad Godesberg nel novembre 1959. Suona corretta la riflessione dell'autore a detta del quale «la SPD aveva [...] compreso che, se un partito vuole veramente rimanere se stesso, deve avere la capacità di comprendere e adattarsi ai mutamenti in atto, mentre i socialisti italiani faticavano ancora ad accettare le logiche del riformismo, essendo ancora legati ai condizionamenti dettati dal filosovietismo degli anni precedenti» ${ }^{21}$. Anche le intuizioni comparative cui Scroccu si richiama per illustrare i simili punti di vista che il gruppo di Raniero Panzieri e Lucio Libertini e la New Left del partito laburista davano al fenomeno del neocapitalismo, costituiscono un forte segno di discontinuità con le precedenti interpretazioni storiche poiché, se è pur vero che il lavoro ha carattere prettamente interno, l'autore dimostra di saper contestualizzare a livello sovra-nazionale l'evoluzione del PSI, offrendo nuovi spunti di ricerca agli studiosi del settore.

${ }^{21}$ Ibidem, pp. 185-186. 


\section{* L'autore}

Jacopo Perazzoli si è laureato in storia presso l'Università degli Studi di Milano con una tesi sul rinnovamento della Socialdemocrazia tedesca nel corso degli anni cinquanta. Dal novembre 2011 è dottorando di ricerca in scienze storiche presso l'Università degli Studi del Piemonte Orientale e dal febbraio 2013 al luglio 2013 è stato visiting student presso la Kingston University di Londra. Il suo progetto di ricerca è dedicato allo studio comparato dell'evoluzione del partito laburista inglese, della SPD e del PSI a cavallo tra gli anni cinquanta. Ha curato il volume Antonio Greppi. Novant'anni di socialismo. Scritti scelti (Milano, Edizioni l'Ornitorinco, 2012) e contribuito alla stesura del saggio La sinistra arancione. Da Milano all'Italia? (Milano, Edizioni l'Ornitorinco, 2012).

URL: < http://www.studistorici.com/progett/autori/\#Perazzoli >

\section{Per citare questo articolo:}

PERAZZOLI, Jacopo, «Recensione: Gianluca SCROCCU, II partito al bivio. II PSI dall'opposizione al governo (19531963), Roma, Carocci, 2011, 358 pp.», Diacronie. Studi di Storia Contemporanea : Le monarchie nell'età dei nazionalismi, 29/12/2013,

URL: < http://www.studistorici.com/2013/12/29/perazzoli_numero_16/ >

\section{Diacronie Studi di Storia Contemporanea $\beta$ www.diacronie.it}

Risorsa digitale indipendente a carattere storiografico. Uscita trimestrale. redazione.diacronie@hotmail.it

Comitato di redazione: Marco Abram - Jacopo Bassi - Luca Bufarale - Alessandro Cattunar - Elisa Grandi - Deborah Paci - Fausto Pietrancosta - Matteo Tomasoni - Luca Zuccolo

Diritti: gli articoli di Diacronie. Studi di Storia Contemporanea sono pubblicati sotto licenza Creative Commons 2.5. Possono essere riprodotti a patto di non modificarne i contenuti e di non usarli per fini commerciali. La citazione di estratti è comunque sempre autorizzata, nei limiti previsti dalla legge. 